\title{
$\mathrm{HCl}$ Engineering: Charting the Way towards Methods and Tools for Advanced Interactive Systems
}

\author{
Jürgen Ziegler \\ University of Duisburg-Essen \\ Duisburg, Germany \\ juergen.ziegler@uni-due.de
}

\author{
José Creissac Campos \\ Universidade do Minho \\ Braga, Portugal \\ jose.campos@di.uminho.pt
}

\author{
Laurence Nigay \\ Univ. Grenoble Alpes, LIG \\ CNRS, LIG, F-38000 Grenoble \\ laurence.nigay@imag.fr
}

\begin{abstract}
This workshop intends to establish the basis of a roadmap addressing engineering challenges and emerging themes in HCI. Novel forms of interaction and new application domains involve aspects that are currently not sufficiently covered by existing methods and tools. The workshop will serve as a venue to bring together researchers and practitioners interested the Engineering of HumanComputer Interaction and in contributing to the definition of a roadmap for the field. The intention is to continue work on the roadmap in follow-up workshops as well as in the IFIP Working Group on User Interface Engineering.
\end{abstract}

\section{WORKSHOP TOPICS AND SCOPE}

Engineering interactive systems is a multidisciplinary endeavor positioned at the intersection of HCI, software engineering, usability engineering, interaction design, visual design and other disciplines..

The field of Human-Computer Interaction Engineering (HCI-E) is concerned with providing methods and tools for the systematic and effective design, development and evaluation of interactive systems in a wide range of application areas. The aim of such methods and tools is twofold: (1) Improve the process of designing, developing and evaluating interactive systems (2) Improve the quality of the user interface of interactive systems, including usability properties and software properties (also called external and internal properties respectively in [1]).

Traditionally, HCI-E methods and tools have mainly addressed standard graphical, mobile, multimodal and multi-device interactive systems, as well as special themes such as safety-critical systems in the medical domain and automotive user interfaces. In recent years, the range of interactive techniques available and their applications has

Permission to make digital or hard copies of part or all of this work for personal or classroom use is granted without fee provided that copies are not made or distributed for profit or commercial advantage and that copies bear this notice and the full citation on the first page. Copyrights for third-party components of this work must be honored. For all other uses, contact the Owner/Author.

Copyright is held by the owner/author(s)

EICS'14, Jun 17-20 2014, Rome, Italy

ACM 978-1-4503-2725-1/14/06.

http://dx.doi.org/10.1145/2607023.2610289 broadened considerably and can be expected to grow even further in the future. While new interaction techniques offer the prospect of improving the usability and user experience of interactive systems, they also pose new challenges for methods and tools that can support their design development and evaluation in a systematic engineeringoriented manner. This is aggravated by the fact that they are increasingly being applied in novel and less understood application domains (e.g., wearable medical devices and invehicle applications).

Examples of innovative interaction techniques with little methodological support so far include:

- $\quad$ tangible and mixed-reality interfaces;

- 3D interaction;

- perceptual interfaces based on computer vision, speech recognition, or the measurement of biosignals;

- visualization techniques and their application to big data, e.g. social media applications.

The techniques mentioned above as well as many other novel forms of interaction involve aspects that are currently not sufficiently covered by existing HCI-E methods/tools such as design spaces, task models, model-based generation of user interfaces, toolkit, evaluation methods. This may require new methods/tools or adaptations/extensions of existing methods/tools.

In addition to emerging interaction techniques, there are remaining HCI-E issues for more established interaction techniques (e.g., mobile interaction, multimodality, collaborative interaction). For instance prototyping techniques and approaches for designing mobile applications was the topic of a workshop at the last MobilHCI conference [3]. While prototyping tools exist for GUI, they require adaptations and extensions to cover the range of issues involved in mobile $\mathrm{HCI}$ and Ubiquitous Computing (e.g., multi-touch gestures, usage of sensors for interaction).

\section{GOALS OF THE WORKSHOP}

The workshop aims at identifying, examining and structuring the engineering challenges related to novel forms of interaction or to emerging themes in HCI due to 
new application domains. An intended outcome of the workshop is an organized overview of engineering challenges and of areas that currently lack systematic method or tool support. These results shall serve as a basis for drafting a roadmap for engineering advanced interactive systems, consolidating, structuring and prioritizing open research questions.

While it is not realistic to cover all methodological and tool-related research questions in depth in a one-day workshop, the intention is to continue work on the roadmap either in follow-up workshops as well as in the context of the IFIP Working Group on User Interface Engineering [2] where external participants are welcome.

\section{FORMAT AND ORGANIZATION}

\section{Soliciting and Selecting Contributions}

We will solicit contributions from the HCI-E related communities and we would be very interested to welcome both researchers and practitioners for a fruitful discussion. To do so we will dispatch the call to the usual channels including announcements in mailing lists, conferences and personal contacts (in particular for practitioners).

Prospective participants should submit a 2-page position paper describing their interests in and views on future engineering methods/tools, as well as related previous work (including their own) where appropriate. At least, each position paper should describe a major engineering-related challenge to be further elaborated at the workshop. We will select participants on the basis of the abstract's quality, their response to the list of issues and the diversity of their backgrounds, aiming thus at an interdisciplinary group. A further criterion will be the stated intention to continue work on elaborating and updating the roadmap after the workshop.

\section{Pre-workshop activities}

We will compile and publish a list of the challenges submitted by the accepted participants on the workshop website prior to the event. The selected presenters will be asked to provide an extended 5-page position paper two weeks before the workshop. These position papers will be circulated in advance to all participants to obtain an understanding of the mutual views and to provide a starting point for the discussion.

\section{Structure and schedule of the workshop}

The workshop will last one day and will comprise brief position statements by the participants followed by a plenary discussion and an initial structuring of the topics. Breakout groups will further refine the different research areas. Based on the reports from the breakout groups, the research field will be further structured with the aim of producing an initial version of a research roadmap for HCI Engineering.

\section{Plan for dissemination}

We will make the workshop results available on the workshop website and plan to publish extended paper versions electronically after the workshop. We also plan to produce a publication, e.g. a journal paper, summarizing and consolidating the contributions in the form of an Engineering HCI roadmap. Furthermore, the results will serve as inputs to future work and discussions in the IFIP Working Group on User Interface Engineering [2].

\section{ORGANIZERS BACKGROUND AND EXPERIENCES}

José C. Campos is an assistant professor at the Department of Informatics of Universidade do Minho, and a member of HASLab / INESC TEC. His main area of research is the verification and validation of interactive computing system. Among other roles, he was general co-chair for EICS 2012 and tutorials co-chair for INTERACT 2011. He was awarded an International Excellence Award by the BCS HCI Group, for his paper on Systematic Analysis of Control Panel Interfaces Using Formal Tools at DSVIS 2008.

Laurence Nigay is a full Professor in Computer Science at Université of Grenoble 1. She is the director of the Engineering Human-Computer Interaction (EHCI) research group of the Grenoble Informatics Laboratory. From 19982004, she was vice-chair of the IFIP working group WG 2.7/13.4 User Interface Engineering. Her research interests include new interaction techniques, Multimodal and Augmented Reality (AR) user interfaces. She has received several scientific awards (including the CNRS Bronze medal in 2002 and the UJF gold medal in 2005) for excellence in her research.

Jürgen Ziegler is a full Professor in Interactive Systems and Interaction Design at the University of Duisburg-Essen. His main areas of research are context-adaptive interaction, recommender systems, information visualization and user interfaces for semantic data. He has served in various functions for all major HCI conferences and has coorganized workshops at CHI, IUI, INTERACT and other venues. Currently, he is also chair of IFIP Working Group 2.7/13.4 User Interface Engineering.

\section{REFERENCES}

1. Gram, C. and Cockton G. Editors. 1996. Design Principles for Interactive Software. Chapman \& Hall.

2. IFIP WG 2.7/13.4 Working Group on User Interface Engineering. http://ui-engineering.org/index.html

3. Workshop on Prototyping to Support the Interaction Designing in Mobile Application Development (PIDMAD 2013) http://hciv.de/pidmad/ 